See discussions, stats, and author profiles for this publication at: https://www.researchgate.net/publication/340475687

\title{
LOFAR 144-MHz follow-up observations of GW170817
}

Preprint · April 2020

\section{CITATIONS}

69 authors, including

Kelly Gourdji

University of Amsterdam

18 PUBLICATIONS 655 CITATIONS

SEE PROFILE

Martin J. Hardcastle

University of Hertfordshire

508 PUBLICATIONS 10,141 CITATIONS

SEE PROFILE
READS

48

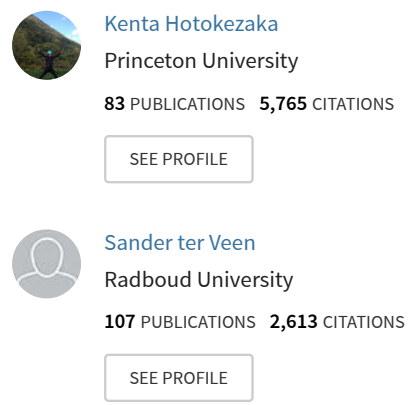

Some of the authors of this publication are also working on these related projects:

The search for radio emission from exoplanets using LOFAR beam-formed observations: View project

Cassini-RPWS View project 


\title{
LOFAR 144-MHz follow-up observations of GW170817
}

\author{
J. W. Broderick, ${ }^{1,2 \star ~ T . ~ W . ~ S h i m w e l l, ~}{ }^{1,3}$ K. Gourdji, ${ }^{4}$ A. Rowlinson, ${ }^{1,4}$ \\ S. Nissanke, ${ }^{5,6}$ K. Hotokezaka, ${ }^{7,8}$ P. G. Jonker, ${ }^{9,10}$ C. Tasse,${ }^{1,12}$ M. J. Hardcastle, ${ }^{13}$
} J. B. R. Oonk, ${ }^{14,1,3}$ R. P. Fender, ${ }^{15}$ R. A. M. J. Wijers, ${ }^{4}$ A. Shulevski, ${ }^{4}$

A. J. Stewart, ${ }^{16}$ S. ter Veen, ${ }^{1}$ V. A. Moss,${ }^{17,1,16}$ M. H. D. van der Wiel,${ }^{1}$

D. A. Nichols, ${ }^{5,18}$ A. Piette, ${ }^{19}$ M. E. Bell,${ }^{20}$ D. Carbone, ${ }^{21}$ S. Corbel,${ }^{22,23}$

J. Eislöffel, ${ }^{24}$ J.-M. Grießmeier, ${ }^{25,23}$ E. F. Keane, ${ }^{26}$ C. J. Law, ${ }^{27}$

T. Muñoz-Darias, ${ }^{28,29}$ M. Pietka, ${ }^{15}$ M. Serylak, ${ }^{30,31}$ A. J. van der Horst, ${ }^{32,33}$

J. van Leeuwen, ${ }^{1,4}$ R. Wijnands, ${ }^{4}$ P. Zarka, ${ }^{34}$ J. M. Anderson, ${ }^{35,36}$

M. J. Bentum,,${ }^{1,37}$ R. Blaauw, ${ }^{1}$ W. N. Brouw,${ }^{38}$ M. Brüggen, ${ }^{39}$ B. Ciardi, ${ }^{40}$

M. de Vos, ${ }^{1}$ S. Duscha, ${ }^{1}$ R. A. Fallows,${ }^{1}$ T. M. O. Franzen, ${ }^{1}$ M. A. Garrett, ${ }^{41,3}$

A. W. Gunst, ${ }^{1}$ M. Hoeft, ${ }^{24}$ J. R. Hörandel,,${ }^{10,6,42}$ M. Iacobelli, ${ }^{1}$ E. Jütte, ${ }^{43}$

L. V. E. Koopmans, ${ }^{38}$ A. Krankowski, ${ }^{44}$ P. Maat, ${ }^{1}$ G. Mann,,${ }^{45}$ H. Mulder,${ }^{1}$

A. Nelles, ${ }^{46,47}$ H. Paas, ${ }^{48}$ M. Pandey-Pommier,${ }^{23,49}$ R. Pekal, ${ }^{50}$ W. Reich, ${ }^{51}$

H. J. A. Röttgering, ${ }^{3}$ D. J. Schwarz, ${ }^{52}$ O. Smirnov, ${ }^{12,30}$ M. Soida, ${ }^{53}$

M. C. Toribio, ${ }^{54}$ M. P. van Haarlem, ${ }^{1}$ R. J. van Weeren, ${ }^{3}$ C. Vocks,${ }^{45}$

O. Wucknitz ${ }^{51}$ and P. Zucca ${ }^{1}$

Affiliations are listed at the end of the paper

Accepted XXX. Received YYY; in original form ZZZ

\begin{abstract}
We present low-radio-frequency follow-up observations of AT 2017gfo, the electromagnetic counterpart of GW170817, which was the first binary neutron star merger to be detected by Advanced LIGO-Virgo. These data, with a central frequency of $144 \mathrm{MHz}$, were obtained with LOFAR, the Low-Frequency Array. The maximum elevation of the target is just 13 :7 when observed with LOFAR, making our observations particularly challenging to calibrate and significantly limiting the achievable sensitivity. On timescales of 130-138 and 371-374 days after the merger event, we obtain $3 \sigma$ upper limits for the afterglow component of 6.6 and $19.5 \mathrm{mJy} \mathrm{beam}^{-1}$, respectively. Using our best upper limit and previously published, contemporaneous higher-frequency radio data, we place a limit on any potential steepening of the radio spectrum between 610 and $144 \mathrm{MHz}$ : the two-point spectral index $\alpha_{144}^{610} \gtrsim-2.5$. We also show that LOFAR can detect the afterglows of future binary neutron star merger events occurring at more favourable elevations.
\end{abstract}

Key words: gravitational waves - radio continuum: stars - stars: neutron 
the Advanced Virgo interferometer (Advanced LIGO-Virgo; Acernese et al. 2015; LIGO Scientific Collaboration et al. 2015) detected gravitational waves (GWs) from the binary neutron star merger GW170817 (Abbott et al. 2017c). The subsequent discovery and initial characterisation of the electromagnetic counterpart, AT 2017gfo (Abbott et al. 2017a and references therein), located in the galaxy NGC 4993 (heliocentric redshift $z=0.00978$; distance $\approx 40 \mathrm{Mpc}$; Hjorth et al. 2017), was truly a landmark event in multimessenger astrophysics.

Following the short gamma-ray burst (sGRB) associated with this event, GRB 170817A (Abbott et al. 2017a,b; Goldstein et al. 2017), radio emission was anticipated as the associated merger outflow interacted with the circum-merger medium. Monitoring the radio emission could therefore provide crucial information on the energetics and geometry of the outflow, as well as the ambient environment. At radio frequencies, telescopes were observing the Advanced LIGOVirgo probability region for GW170817 within 29 min post merger (Callister et al. 2017a), and subsequent monitoring of AT 2017gfo resulted in an initial radio detection 16 days after the event (Abbott et al. 2017a; Hallinan et al. 2017). Further monitoring, predominantly at frequencies between 0.6 and $15 \mathrm{GHz}$, has since taken place (e.g. Alexander et al. 2017, 2018; Mooley et al. 2018a,b,c; Margutti et al. 2018; Dobie et al. 2018; Troja et al. 2018, 2019; Corsi et al. 2018; Resmi et al. 2018). At these frequencies, a general picture emerged in which the radio light curve was first observed to steadily rise, before it turned over and began a more rapid decay. Using a compilation of $0.6-10 \mathrm{GHz}$ radio data from 17-298 days post merger, Mooley et al. (2018c) derived both a fitted time for the radio peak of $174_{-6}^{+9}$ days and a fitted 3 - GHz peak flux density of $98_{-9}^{+8} \mu \mathrm{Jy}$ (also see similar analyses in Dobie et al. 2018 and Alexander et al. 2018). The fitted radio spectral index $\alpha^{1}$ from this study is $-0.53 \pm$ 0.04 , consistent with broadband spectral indices determined using radio, optical and $\mathrm{X}$-ray data at various epochs, where the typical value is approximately -0.58 (e.g. Margutti et al. 2018; Troja et al. 2018, 2019; Alexander et al. 2018; Hajela et al. 2019). Mooley et al. (2018c) also found power-law dependencies for the rise and decay phases of approximately $t^{0.8}$ and $t^{-2.4}$, respectively, where $t$ is the time since the merger. Within the associated uncertainties, these results are consistent with the broadband evolution of AT 2017gfo (e.g. Alexander et al. 2018; Lamb et al. 2019; Troja et al. 2019; Hajela et al. 2019).

Two competing models emerged to explain the radio light curve: either the jet successfully broke through the surrounding cocoon of ejected material (also known as a 'structured' jet) but was observed off-axis, or the jet was 'choked' by the cocoon, in which it deposited all of its energy (e.g. Troja et al. 2017; Kasliwal et al. 2017). The observed evolution of the radio light curve, and very-long-baseline interferometric measurements of both apparent superluminal motion and a sufficiently compact apparent source size, confirmed that a jet was successfully launched for GW170817 with opening angle $<5^{\circ}$ and observed from a viewing angle

1 We use the convention that $S_{v} \propto v^{\alpha}$, where $S_{v}$ is the flux density at frequency $v$. of approximately $15-20^{\circ}$ (Alexander et al. 2018; Mooley et al. 2018b,c; Ghirlanda et al. 2019).

Although predicted faint flux density levels and slow light curve rise times (e.g. Hotokezaka et al. 2016) may make late-time, low-radio-frequency $(\lesssim 200 \mathrm{MHz})$ detections challenging, such flux density measurements can help to discriminate between competing models for the radio emission following a compact binary merger. In addition, the current generation of low-frequency aperture arrays have rapid electronic beam steering, as well as very large fields of view that can cover at minimum a significant fraction of the Advanced LIGO-Virgo probability region for a GW event. Therefore, there is the interesting potential to use low-frequency aperture arrays to search for prompt, coherent radio emission from a compact binary merger (e.g. Callister et al. 2019; also see Obenberger et al. 2014, Yancey et al. 2015, Kaplan et al. 2015, 2016, Chu et al. 2016, Anderson et al. 2018, Rowlinson \& Anderson 2019, James et al. 2019 and Rowlinson et al. 2019).

At low frequencies, GW170817 was followed up with both the first station of the Long Wavelength Array (LWA1; Ellingson et al. 2013) and the Murchison Widefield Array (MWA; Tingay et al. 2013). Details of the LWA1 observations can be found in Callister et al. (2017a,b,c) and Abbott et al. (2017a), including the aforementioned observation 29 min post merger, as well as additional observations up to approximately 13 days after the event. Similarly, details of the MWA observations, occurring 0.8-4.9 days post merger, can be found in Kaplan et al. (2017a,b), Abbott et al. (2017a) and Andreoni et al. (2017). At the position of NGC 4993, 26- and 45-MHz LWA1 observations approximately $8 \mathrm{~h}$ after the event yielded $3 \sigma$ upper limits of 200 and $100 \mathrm{Jy}$, respectively, for persistent emission (Callister et al. 2017c). At $185 \mathrm{MHz}, 0.8$ days post merger, the $3 \sigma$ MWA upper limit was $51 \mathrm{mJy}^{\text {beam }^{-1}}$, albeit with only 40 of the 128 tiles operational at the time (Kaplan et al. 2017b; Andreoni et al. 2017).

In this paper, we present late-time (130-138 and 371374 days post merger), low-radio-frequency follow-up observations of AT $2017 \mathrm{gfo}$, obtained with the high-band antennas (HBA) of the Low-Frequency Array (LOFAR; van Haarlem et al. 2013). In Section 2, we describe these observations, and how the data were calibrated and imaged. Our results are presented in Section 3, which is followed by a discussion in Section 4 on the additional constraints that our $144-\mathrm{MHz}$ upper limits place on the properties of the radio emission from GW170817, as well as future prospects for LOFAR when observing new GW events. We then conclude in Section 5. All uncertainties reported in this paper are quoted at the 68 per cent confidence level.

\section{LOFAR OBSERVATIONS AND DATA REDUCTION}

Table 1 presents a log of the two observing runs. In both cases, we used the 'HBA Dual Inner' configuration, with 24 core stations and either 13 or 14 Dutch remote stations. Because the location of AT 2017gfo on the sky (Dec $=-23^{\circ} .4$ ) is very far south relative to LOFAR (latitude of core $=52^{\circ} .9$ $\mathrm{N}$ ), both runs were split into $4 \times 2$-h observations on separate days, each centred as closely to transit as possible, 
so as to maximize the elevation of the target. Nonetheless, the maximum elevation as viewed from the LOFAR core is only 13.7 , which significantly affected the sensitivity that we could achieve due to the small projected station area, as well as making our observations far more susceptible to ionospheric effects. Both sets of observations comprised 380 $\times 195.3-\mathrm{kHz}$ sub-bands that spanned the frequency range 115-189 MHz, although in this study we made use of the most sensitive part of the bandpass between $120-168 \mathrm{MHz}$ (246 sub-bands). All 2-h observations were preceded by a 10-min scan of the flux density calibrator 3C 295.

Preprocessing consisted of flagging and averaging (in time and/or frequency) steps. The former made use of AOFLAGGER (Offringa et al. 2010; Offringa, van de Gronde \& Roerdink 2012a; Offringa, de Bruyn \& Zaroubi 2012b), with 14.3 and 15.9 per cent of data flagged per sub-band on average for Runs 1 and 2, respectively. After preprocessing, the temporal and frequency resolutions for Run 1 were $1 \mathrm{~s}$ and $12.2 \mathrm{kHz}$ (i.e. 16 channels per sub-band), respectively; the corresponding values were $4 \mathrm{~s}$ and $48.8 \mathrm{kHz}$ (i.e. 4 channels per sub-band), respectively, for Run 2 . These differing resolutions between Runs 1 and 2 were due to the fact that Run 2 was obtained as part of a larger LOFAR GW follow-up project with different preprocessing settings (Gourdji et al. in prep.). In principle, however, Run 2 still had sufficiently fine temporal and frequency resolutions to permit proper calibration, as was the case for Run 1 (e.g. see Shimwell et al. 2017, 2019).

The reduction steps after preprocessing were directionindependent calibration, followed by direction-dependent calibration and imaging. A detailed description of the procedure, which was developed for the LOFAR Two-metre Sky Survey (LoTSS), can be found in Shimwell et al. (2017, 2019). The direction-independent pipeline removed the effects outlined in de Gasperin et al. (2019), following the procedure described in van Weeren et al. (2016) and Williams et al. (2016); it made use of the BBS (Pandey et al. 2009) and DPPP (van Diepen \& Dijkema 2018) software packages. ${ }^{2}$ We used a model for 3C 295 that is consistent with the Scaife \& Heald (2012) flux density scale. The sky model used to calibrate the target data was derived from the TIFR Giant Metrewave Radio Telescope (GMRT; Swarup 1991) Sky Survey First Alternative Data Release (TGSS ADR1; Intema et al. 2017).

The direction-dependent step made use of KMS (Tasse 2014a,b; Smirnov \& Tasse 2015) and DDFACET (Tasse et al. 2018) for calibration and imaging, respectively. ${ }^{3}$ The calibrated data per 2-h observation comprised 25 blocks of 10 sub-bands each; the highest-frequency block had four empty sub-bands because we used 246 sub-bands in total. The final temporal and frequency resolutions were $8 \mathrm{~s}$ and $97.7 \mathrm{kHz}$ (i.e. 2 channels per sub-band), respectively, and the central frequency was $144 \mathrm{MHz}$.
Table 1. An observing log for our LOFAR observations of AT 2017gfo. Both observations had a frequency range of 120-168 $\mathrm{MHz}$, and a central frequency of $144 \mathrm{MHz}$.

\begin{tabular}{ccc}
\hline & \multicolumn{2}{c}{ Observing run } \\
& 1 & 2 \\
\hline Date & 2017 December 25, 27, & 2018 August \\
& 28,2018 January 2 & $23-26$ \\
Days post merger & $130-138$ & $371-374$ \\
RMS noise level & $2.1 \pm 0.6$ & $6.2 \pm 1.9$ \\
$\left(\right.$ mJy beam $\left.{ }^{-1}\right)$ & & \\
Observation IDs & $632609-$ & $664578-$ \\
& $632637^{\mathrm{a}}$ & $664604^{\mathrm{b}}$ \\
\hline
\end{tabular}

a IDs increase in steps of 4 .

b IDs increase in alternate steps of 2 and 6 .

\section{RESULTS}

In the left panel of Figure 1, we show our LOFAR map from Run 1, centred on the position of AT 2017gfo. The corresponding map from Run 2 has a lower dynamic range. When imaging, we had to take into account a primary beam that is very elongated in the north-south direction (FWHM $\approx 23^{\circ} .6 \times 4^{\circ} .8$ ). The angular resolution is as good as $15 \operatorname{arcsec}$, with full details of the synthesised beams given in Figure 1.

At the angular resolution of our observations, any emission from AT 2017gfo and the active galactic nucleus of NGC 4993 could be partially blended, depending on the brightnesses of the sources. In neither of our maps is this potentially blended emission detected, nor are there detections at the two separate sets of coordinates (right panels of Figure 1). In terms of establishing upper limits for the target flux density, the largest source of uncertainty is related to a standard frequency-dependent error in the flux density scale (see references on LOFAR calibration in Section 2 for further details), which we have corrected for, to first order, by bootstrapping to TGSS within a $1^{\circ}$ radius from the position of AT 2017gfo. We used an integrated flux density to peak flux density ratio of $\leq 1.5$ in TGSS to restrict the bootstrapping procedure to sources within the search radius that were not too extended, while retaining a sufficient number of sources for a reasonable statistical comparison. A small adjustment was also made for the slightly different central frequency of TGSS $(147.5 \mathrm{MHz})$, assuming a canonical spectral index of -0.7 . Source finding in the LOFAR maps made use of PYBDSF (Mohan \& Rafferty 2015).

The multiplicative correction factors to apply to our LOFAR maps were found to be $1.3 \pm 0.3$ and $3.3 \pm 1.0$ for Runs 1 and 2, respectively. Moreover, in this region of sky, we found that the TGSS flux density scale is consistent to within about 10 per cent on average with the corresponding scale from the Galactic and Extragalactic All-sky Murchison Widefield Array Survey (GLEAM; Hurley-Walker et al. 2017). Using appropriate error propagation, we combined

\footnotetext{
2 https://github.com/lofar-astron/prefactor using commit dd68c57.

3 In particular, we used version 2.2 of DDF-PIPELINE, which can be found at https://github.com/mhardcastle/ddf-pipeline.
} 

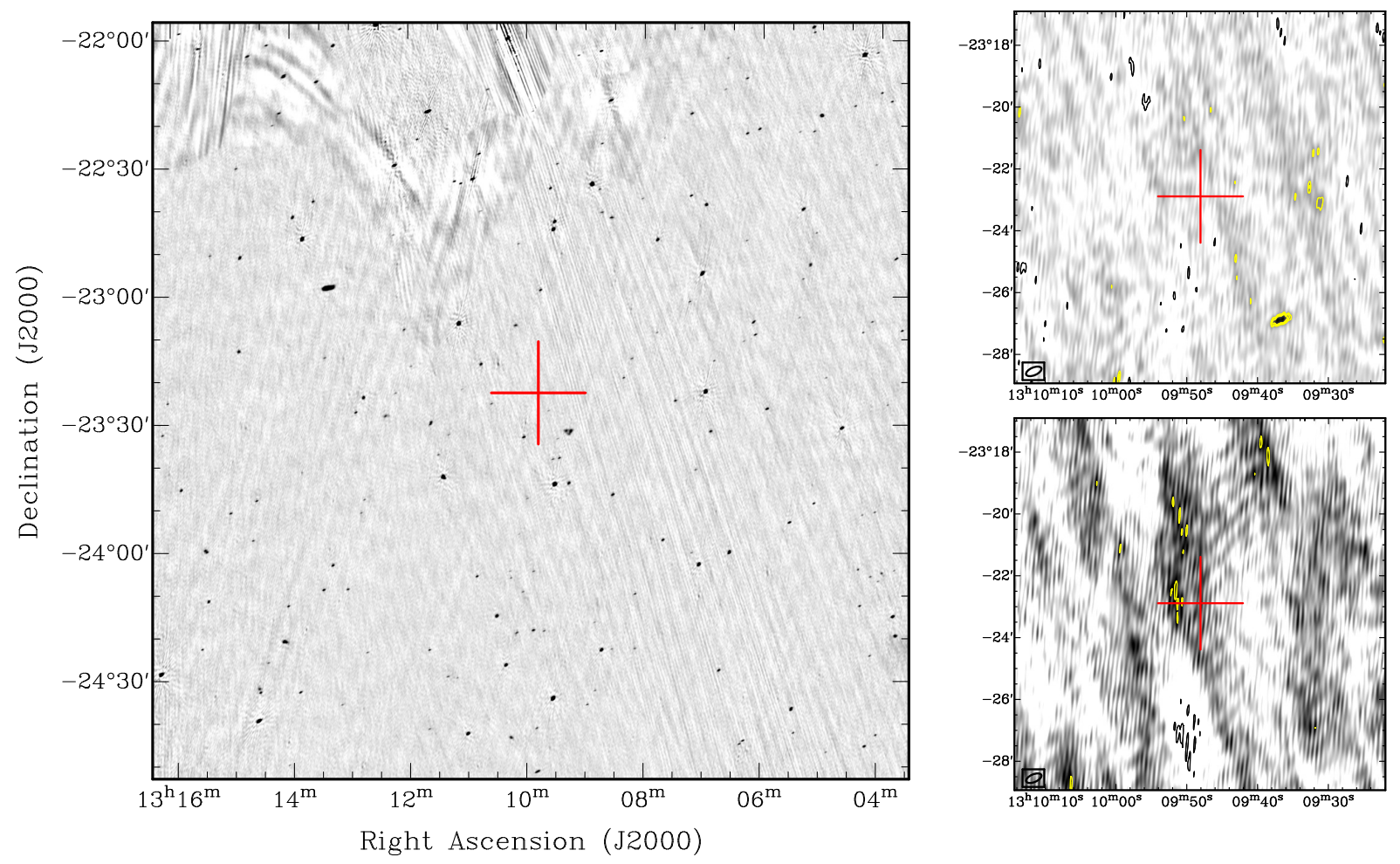

Figure 1. The left panel is our LOFAR 144-MHz image of a field centred on AT 2017gfo, obtained from the first set of observations 130-138 days post merger. We show a subsection with dimensions $3^{\circ} \times 3^{\circ}$; the crosshairs mark the position of AT 2017gfo. The two panels on the right (with the same grey-scale contrast scheme) show a more zoomed-in view $\left(0.2^{\circ} \times 0.2^{\circ}\right)$, where Run 1 is the top panel and Run 2 the bottom panel. The RMS noise levels in the vicinity of the position of AT 2017gfo are approximately 2.1 and $6.2 \mathrm{mJy}$ beam $^{-1}$ for Runs 1 and 2 , respectively; the contour scheme is $-3 \sigma$ (black) and $(3,4,5) \times \sigma$ (yellow). The restoring beam, chosen to be the same for both runs (32 arcsec $\times 15$ arcsec; beam position angle $-67^{\circ}$ measured north through east) is shown in the bottom left-hand corner of these two panels.

each bootstrapping uncertainty with a 10 per cent absolute flux density calibration uncertainty. After doing this, the RMS noise levels $(\sigma)$ are $2.1 \pm 0.6$ and $6.2 \pm 1.9 \mathrm{mJy}$ beam $^{-1}$ for Runs 1 and 2 , respectively, in the vicinity of the target position. To obtain $3 \sigma$ upper limits that are correct to first order, we then combined each RMS value with its respective uncertainty, in quadrature, before multiplying by three. Therefore, our $3 \sigma$ upper limits for AT 2017gfo (and NGC 4993) are 6.6 and $19.5 \mathrm{mJy}^{\text {beam }}{ }^{-1}$ for Runs 1 and 2, respectively.

As is apparent in Figure 1, the dynamic range is limited in the north and north-east of the map, but the image quality nearer the centre of the map is relatively unaffected. Moreover, to first order, there is a good correspondence between the source morphologies and positions in the LOFAR and TGSS images. ${ }^{4}$ While a detailed comparison is beyond the scope of this paper, it is worth noting that the noise level reported above for Run 1 is the deepest value obtained thus far in the literature for LOFAR interferometric observations significantly south of the celestial equator. Unfortunately, however, the noisier map from the second run does not share the same overall consistency with TGSS, and the correction factor reported above for Run 2 is unusually large. Poorer ionospheric conditions are likely to be a contributing factor.

4 The TGSS image archive can be found at https://vo.astron. nl/tgssadr/q_fits/imgs/info.
The upper limit from this run should therefore be viewed with caution, although we note that this does not affect any subsequent discussion in this paper.

\section{DISCUSSION}

\subsection{Low-frequency constraints on the radio spectrum of AT 2017gfo}

We now discuss the additional constraints that can be placed on the radio spectrum of AT $2017 \mathrm{gfo}$, as well as NGC 4993. First, Resmi et al. (2018) presented 610- and $1390-\mathrm{MHz}$ GMRT flux densities for both AT 2017gfo and the nucleus of NGC 4993. In the case of NGC 4993, the flux densities are relatively faint, and the radio spectrum relatively flat. After averaging the reported flux densities using inverse-variance weighting, we find that $\bar{S}_{610} \approx 0.99 \mathrm{mJy}, \bar{S}_{1390} \approx 0.78 \mathrm{mJy}$, and the mean two-point spectral index $\bar{\alpha}_{610}^{1390} \approx-0.29$. Therefore, our best $3 \sigma$ upper limit at $144 \mathrm{MHz}$ only provides a very weak, additional constraint of $\alpha_{144}^{610} \gtrsim-1.3$.

In the case of AT $2017 \mathrm{gfo}$, our first set of observations is either bookended by or close in time to a selection of the 610- and 1390-MHz GMRT observations, as well as Australia Telescope Compact Array (ATCA; Frater, Brooks \& Whiteoak 1992; Wilson et al. 2011) observations carried out at 5500 and $9000 \mathrm{MHz}$ (Dobie et al. 2018). For the 1390-MHz observations that bracketed our data, we averaged the corre- 

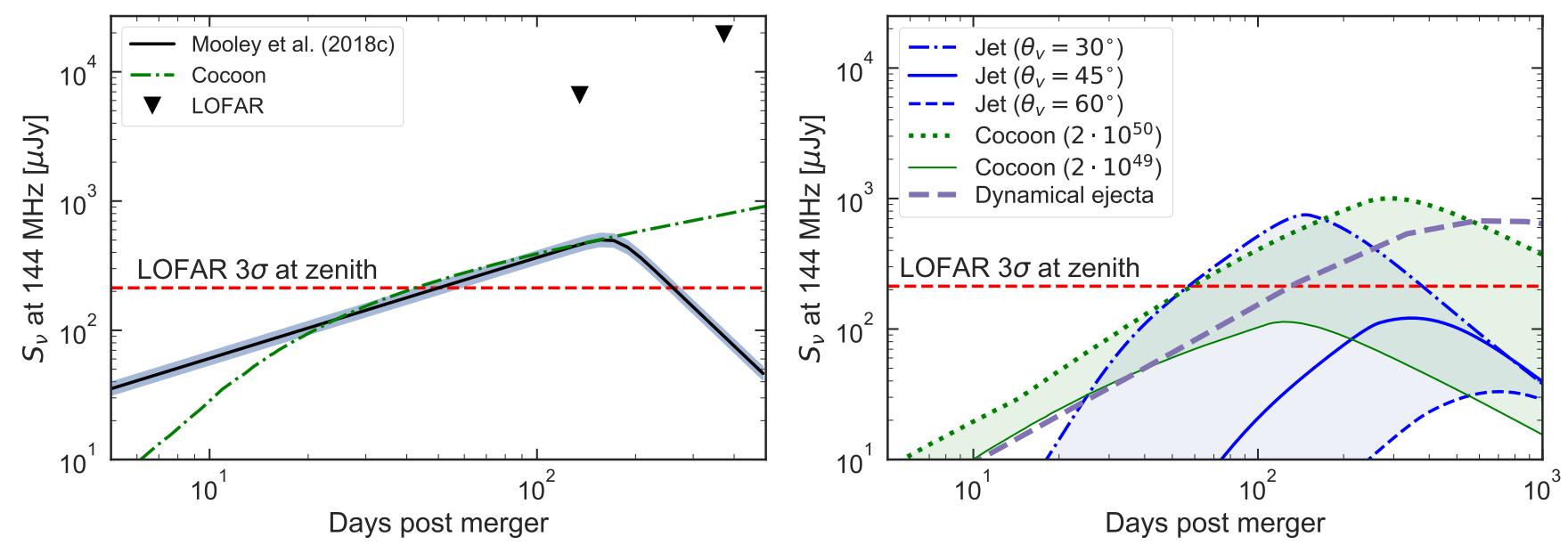

Figure 2. Afterglow light curves at $144 \mathrm{MHz}$ for GW170817 (left panel) and an example future event at a distance of $100 \mathrm{Mpc}$ (right panel). In the left panel, the solid line with surrounding shading is the light curve at $144 \mathrm{MHz}$ extrapolated from the observations at higher frequencies with $\alpha=-0.53 \pm 0.04$ (Mooley et al. 2018c). The triangles are our $3 \sigma$ LOFAR upper limits, and the median $3 \sigma$ sensitivity of LOFAR for routine 8-h observations for declinations at or near zenith (see Section 4.2) is depicted as a dashed horizontal line. The dash-dotted line is an analytic cocoon model described in Mooley et al. (2018a) with circum-merger density $n=10^{-4} \mathrm{~cm}^{-3}$, and microphysical parameters $\epsilon_{e}=0.1, \epsilon_{B}=0.01$, and $p=2.1$. In the right panel, $n$ is chosen to be $0.01 \mathrm{~cm}^{-3}$, and the microphysical parameters are $\epsilon_{e}=0.1, \epsilon_{B}=0.01$, and $p=2.2$. We show a structured jet model for various viewing angles, details of which are given in Section 4.2. For the cocoon model in this panel, we show two different kinetic energies of $2 \times 10^{49}$ and $2 \times 10^{50}$ erg. A dynamical ejecta light curve (model ' $\mathrm{DNS}_{\mathrm{m}}$ ') taken from Hotokezaka et al. (2016) is also shown.

sponding flux densities using inverse-variance weighting; we also did this for the ATCA data, but using the $7250-\mathrm{MHz}$ flux densities with a correction factor applied from Mooley et al. (2018c). We can then combine these (averaged) flux densities with our LOFAR $3 \sigma$ upper limit from Run 1 to calculate approximate constraints on a number of twopoint spectral indices, roughly $125-150$ days post merger. The constraints are $\alpha_{144}^{610} \gtrsim-2.5, \alpha_{144}^{1390} \gtrsim-1.8$, and $\alpha_{144}^{7250} \gtrsim$ -1.2 . These limits are still significantly steeper than the fitted $0.6-10 \mathrm{GHz}$ radio spectral index of $-0.53 \pm 0.04$ as determined by Mooley et al. (2018c) (see Section 1). Therefore, we can only rule out that the radio spectrum of AT $2017 \mathrm{gfo}$ does not steepen below $610 \mathrm{MHz}$ to an extreme degree.

Coherent radio emission can result in an ultra-steep spectrum component that is only observable at low frequencies. An overview of the physical mechanisms by which coherent radio emission may arise from a compact binary merger was given by Rowlinson \& Anderson (2019). In the case of GW170817, and on a time-scale of 130-138 days after the merger, there are two immediate considerations. First, two-point spectral indices similar to those calculated above would have to be flatter than our lower limits. Secondly, a long-lived neutron star merger remnant would be required. Whether such a stable remnant was, and remains, present, or collapsed to a black hole on a much shorter time-scale, has been the subject of considerable discussion in the literature (e.g. Metzger, Thompson \& Quataert 2018; Ai et al. 2018; Yu, Liu \& Dai 2018; Radice et al. 2018b; Piro et al. 2019; Gill, Nathanail \& Rezzolla 2019; Yang et al. 2019).

\subsection{Forecasts for future LOFAR observations of GW events}

The predicted afterglow light curve of GW170817 at 144 $\mathrm{MHz}$ is shown in the left panel of Figure 2. Here, we ex- trapolate the light curve at higher radio frequencies using a spectral index of $-0.53 \pm 0.04$ (Mooley et al. 2018c), corresponding to the assumption that both the characteristic synchrotron frequency, $v_{\mathrm{m}}$, and self-absorption frequency, $v_{\mathrm{a}}$, are lower than $144 \mathrm{MHz} .{ }^{5}$ Indeed, following Hotokezaka et al. (2016), we find $v_{\mathrm{a}} \leq 36 \mathrm{MHz}$ (for circum-merger density $n \leq 0.01 \mathrm{~cm}^{-3}$, kinetic energy $E=10^{49} \mathrm{erg}$, fraction of internal energy given to the electrons $\epsilon_{e}=0.1$, fraction of internal energy contained in the magnetic field $\epsilon_{B}=0.01$, power-law index of the electron distribution $p=2.2$, and the initial velocity of the ejecta in units of the speed of light $\beta_{0}=1$; see discussion later in this section), which is well below the LOFAR HBA observing band. The aforementioned competing cocoon model, which has now been ruled out (see Section 1) is also included. We use the cocoon model described in Mooley et al. (2018a): we use the kinetic energy distribution $E(>\gamma \beta)=2 \times 10^{51}(\gamma \beta)^{-5}$ with $\gamma_{\max }=3.5$, where $\gamma$ and $\beta$ are the Lorentz factor and velocity, respectively.

While our LOFAR upper limits lie well above the two curves, we can consider the hypothetical scenario of LOFAR late-time flux density measurements had an event similar to GW170817 been much further north on the sky. LOFAR can achieve a median noise level of approximately $70 \mu \mathrm{Jy}_{\text {beam }}{ }^{-1}$ in routine 8-h observations, with $48 \mathrm{MHz}$ bandwidth centred at $144 \mathrm{MHz}$, for declinations at or near zenith (Shimwell et al. 2019). Considering the radio light curve fitting in Mooley et al. (2018c) and also Alexander et al. (2018) (in addition, see Dobie et al. 2018), the peak flux density at $144 \mathrm{MHz}$ would be predicted to be at approximately 7-9.5 times the median LOFAR sensitivity level (see left panel of Figure 2).

${ }^{5}$ If this assumption fails, then the predicted flux density at 144 $\mathrm{MHz}$ is lower than that in Figure 2, and the peak of the light curve may be delayed; see e.g. Nakar \& Piran (2011). 
Assuming that any uncertainties arising from a host galaxy contribution were negligible, this would have then allowed us to determine whether a single-power-law radio spectrum also held at low frequencies, or whether there were indications of spectral turnover. In the absence of spectral turnover, we would have also been able to discriminate at late times between competing models of the radio afterglow.

GW170817 occurred relatively close by, and with a circum-merger density below average. It is beyond the scope of this paper to consider a comprehensive range of possible future compact binary mergers and their potential detectability with LOFAR. However, for illustrative purposes, let us now consider a more distant binary neutron star merger at $100 \mathrm{Mpc}$ (i.e. about halfway to the Advanced LIGO design sensitivity horizon; Abbott et al. 2016). As is shown in the right panel of Figure 2, the afterglows of jets, cocoons, and dynamical ejecta of such future merger events can be observed by LOFAR in certain cases if $n \gtrsim 0.01 \mathrm{~cm}^{-3}$. Note that observations of the afterglows of sGRBs show that 30-70 per cent of these events occur in environments where the density of the interstellar medium is $\gtrsim 0.01 \mathrm{~cm}^{-3}$ (Fong et al. 2015). The relevant microphysical parameters are $\epsilon_{e}=$ $0.1, \epsilon_{B}=0.01$, and $p=2.2$ (see Hotokezaka et al. 2016 for further details). We show a structured jet model, which has a uniform jet core up to a certain opening angle, and the energy and initial Lorentz factor decrease with angle as a power law. The kinetic energy of the jet core is $10^{49}$ erg and the initial half-opening angle is $0.05 \mathrm{rad}$, with which the light curve is consistent with the observed features of the GW170817 afterglow (Hotokezaka et al. 2019). We find that LOFAR can detect the afterglows of off-axis jets similar to GW170817 when the viewing angle is less than approximately $40^{\circ}$. The cumulative fraction of merger events detected by Advanced LIGO-Virgo with such a viewing angle is expected to be approximately one half (Nissanke, Kasliwal \& Georgieva 2013).

In the right panel of Figure 2, we use a dynamical ejecta light curve (model ' $\mathrm{DNS}_{\mathrm{m}}$ ') taken from Hotokezaka et al. (2016). The dynamical ejecta may be partly responsible for the kilonova emission at optical and infrared wavelengths, but are unlikely to be the major component in terms of mass. However, since this component is faster than the disc outflow, i.e. the afterglow is brighter, we also consider the dynamical ejecta here.

In this discussion, we have assumed that the host galaxy flux density is negligible at LOFAR frequencies. This will not always be the case. Future GW events that are followed up by LOFAR will include an observation at roughly one week post merger, when early persistent emission and late-time afterglow emission are negligible. This observing strategy provides a comparison image to enable identification of the afterglow, but also a constraint on any host galaxy emission at the location of the GW event.

Future LOFAR observations will be particularly important to determine or constrain $v_{\mathrm{a}}$, which can be above 144 $\mathrm{MHz}$ (i.e. significantly higher than our calculation earlier in this section) and is sensitive to the velocity of the outflow (e.g. Nakar \& Piran 2011). Measuring $v_{\mathrm{a}}$ will enable us to break the degeneracy between the model parameters, leading to a better estimate of the velocity and kinetic energy of the outflow. Not only will such measurements provide us with a better understanding of the afterglow, but will also help constrain the neutron star equation of state if the afterglow of the dynamical ejecta is detected (e.g. Radice et al. 2018a).

\section{CONCLUSIONS AND FUTURE WORK}

In this paper, we presented LOFAR follow-up observations of the compact binary merger event GW170817, which was detected by Advanced LIGO-Virgo. Our conclusions are as follows.

(i) In two sets of $4 \times 2$-h observations, occurring 130 138 and $371-374$ days post merger, we determined $3 \sigma$ upper limits of 6.6 (Run 1) and 19.5 (Run 2) $\mathrm{mJy}_{\text {beam }}{ }^{-1}$ for the $144-\mathrm{MHz}$ flux density of the electromagnetic counterpart, AT 2017gfo.

(ii) Using previously published GMRT and ATCA flux densities at higher radio frequencies, we placed constraints on a number of two-point spectral indices for both AT 2017gfo, and the host galaxy NGC 4993, about 4.5 months post merger. In particular, for AT 2017gfo, $\alpha_{144}^{610} \gtrsim$ -2.5 . The presence of ultra-steep-spectrum coherent radio emission at low frequencies would necessitate a long-lived neutron star remnant.

(iii) We showed that, for declinations at or near zenith, LOFAR will be able to detect various possible radio afterglows for a subset of future merger events.

(iv) We also demonstrated that it is possible to obtain images with LOFAR significantly south of the celestial equator, albeit a factor of about 1.5 dex less sensitive and at an angular resolution 2.5-5.3 times coarser than what is achievable at or near zenith, in this particular case. If LoTSS were to be extended below the celestial equator, with an angular resolution at or near the usual target value of $6 \operatorname{arcsec}$ (Shimwell et al. 2017, 2019), this would allow high-resolution, lowfrequency sky models to be developed at declinations that will be readily accessible with the first phase of the lowfrequency component of the Square Kilometre Array (i.e. SKA1-LOW). For example, Hale et al. (2019) recently presented LOFAR HBA observations of the XMM Large-Scale Structure (XMM-LSS) field, which is centred at a declination of $-4.5^{\circ}$. The angular resolution of their map is 8.5 $\operatorname{arcsec} \times 7.5$ arcsec, and the RMS noise level at the centre of the map is $280 \mu \mathrm{Jy}_{\text {beam }}{ }^{-1}$.

Further LOFAR follow-up is planned for binary neutron star and black hole - neutron star mergers that are detected in the current Advanced LIGO-Virgo observing run ('O3'). Follow-up will occur not only on the time-scales investigated in this paper, but also on time-scales as short as several minutes once an alert is received, using the LOFAR responsive telescope mode (see Rowlinson \& Anderson 2019 for a review of the current rapid-response capabilities of a selection of low-frequency radio facilities, including the MWA and LWA). We can therefore expect to obtain further insight into the role that low-radio-frequency data will play in understanding the physical processes that occur following a compact binary merger containing at least one neutron star. 


\section{ACKNOWLEDGEMENTS}

We thank the referee for a number of helpful suggestions that improved the content and presentation of this paper. We also thank the ASTRON Radio Observatory for setting up and scheduling the observations described in this paper, as well as preprocessing the data. Additionally, we are grateful to Ben Stappers for providing very useful feedback on an earlier version of this manuscript.

This paper is based on data obtained with the International LOFAR Telescope (ILT) under project codes DDT9_002 and LT10_013. LOFAR (van Haarlem et al. 2013) is the Low Frequency Array designed and constructed by ASTRON. It has observing, data processing, and data storage facilities in several countries, that are owned by various parties (each with their own funding sources), and that are collectively operated by the ILT foundation under a joint scientific policy. The ILT resources have benefitted from the following recent major funding sources: CNRS-INSU, Observatoire de Paris and Université d'Orléans, France; BMBF, MIWF-NRW, MPG, Germany; Science Foundation Ireland (SFI), Department of Business, Enterprise and Innovation (DBEI), Ireland; NWO, The Netherlands; The Science and Technology Facilities Council, UK; Ministry of Science and Higher Education, Poland.

The LOFAR direction-independent calibration pipeline (https://github.com/lofar-astron/prefactor) was deployed by the LOFAR e-infragroup on the Dutch National Grid infrastructure with support of the SURF Co-operative through grants e-infra170194, e-infra180087 and e-infra180169 (Mechev et al. 2017). The LOFAR direction-dependent calibration and imaging pipeline (http: //github.com/mhardcastle/ddf-pipeline/) was run on computing clusters at Leiden Observatory and the University of Hertfordshire, which are supported by a European Research Council Advanced Grant [NEWCLUSTERS321271] and the UK Science and Technology Funding Council $[\mathrm{ST} / \mathrm{P} 000096 / 1]$.

PGJ acknowledges funding from the European Research Council under ERC Consolidator Grant agreement no. 647208. SC acknowledges funding support from the UnivEarthS Labex program of Sorbonne Paris Cité (ANR-10LABX-0023 and ANR-11-IDEX-0005-02). TMD acknowledges support from grants AYA2017-83216-P and RYC2015-18148. JvL acknowledges funding from Vici research programme 'ARGO' with project number 639.043.815, financed by the Netherlands Organisation for Scientific Research (NWO). MB acknowledges support by the Deutsche Forschungsgemeinschaft (DFG, German Research Foundation) under Germany's Excellence Strategy - EXC 2121 "Quantum Universe" - 390833306.

This research has made use of the VizieR catalogue access tool, CDS, Strasbourg, France (DOI: $10.26093 / \mathrm{cds} /$ vizier). The original description of the VizieR service was published in A\&AS 143, 23 (Ochsenbein, Bauer \& Marcout 2000). This research has made use of the NASA/IPAC Extragalactic Database (NED), which is operated by the Jet Propulsion Laboratory, California Institute of Technology, under contract with the National Aeronautics and Space Administration. This research has made use of NASA's Astrophysics Data System Bibliographic Services. This project also made use of KVIS (Gooch 1995), TOP-
CAT (Taylor 2005), NUMPY (Oliphant 2006) and MATPLOTLIB (Hunter 2007).

\section{REFERENCES}

Abbott B. P. et al., 2016, LRR, 19, 1

Abbott B. P. et al., 2017a, ApJ, 848, L12

Abbott B. P. et al., 2017b, ApJ, 848, L13

Abbott B. P. et al., 2017c, Phys. Rev. Lett., 119, 161101

Acernese F. et al., 2015, Class. Quantum Grav., 32, 024001

Ai S., Gao H., Dai Z.-G., Wu X.-F., Li A., Zhang B., Li M.-Z., 2018, ApJ, 860, 57

Alexander K. D. et al., 2017, ApJ, 848, L21

Alexander K. D. et al., 2018, ApJ, 863, L18

Anderson M. M. et al., 2018, ApJ, 864, 22

Andreoni I. et al., 2017, PASA, 34, e069

Callister T. et al., 2017a, GCN, 21680

Callister T. et al., 2017b, GCN, 21848

Callister T. et al., 2017c, GCN, 21975

Callister T. A. et al., 2019, ApJ, 877, L39

Chu Q., Howell E. J., Rowlinson A., Gao H., Zhang B., Tingay S. J., Boër M., Wen L., 2016, MNRAS, 459, 121

Corsi A. et al., 2018, ApJ, 861, L10

de Gasperin F. et al., 2019, A\&A, 622, A5

Dobie D. et al., 2018, ApJ, 858, L15

Ellingson S. W. et al., 2013, IEEE Trans. Ant. Prop., 61, 2540

Fong W., Berger E., Margutti R., Zauderer B. A., 2015, ApJ, 815, 102

Frater R. H., Brooks J. W., Whiteoak J. B., 1992, J. Electr. Electron. Eng. Aust., 12, 103

Ghirlanda G. et al., 2019, Science, 363, 968

Gill R., Nathanail A., Rezzolla L., 2019, ApJ, 876, 139

Goldstein A. et al., 2017, ApJ, 848, L14

Gooch R., 1995, in Shaw R. A., Payne H. E., Hayes J. J. E., eds, ASP Conf. Ser. Vol. 77, Astronomical Data Analysis Software and Systems IV. Astron. Soc. Pac., San Francisco, p. 144

Hajela A. et al., 2019, ApJ, 886, L17

Hale C. L. et al., 2019, A\&A, 622, A4

Hallinan G. et al., 2017, Science, 358, 1579

Hjorth J. et al., 2017, ApJ, 848, L31

Hotokezaka K., Nissanke S., Hallinan G., Lazio T. J. W., Nakar E., Piran T., 2016, ApJ, 831, 190

Hotokezaka K., Nakar E., Gottlieb O., Nissanke S., Masuda K., Hallinan G., Mooley K. P., Deller A. T., 2019, Nature Astronomy, 3, 940

Hunter J. D., 2007, Comput. Sci. Eng., 9, 90

Hurley-Walker N. et al., 2017, MNRAS, 464, 1146

Intema H. T., Jagannathan P., Mooley K. P., Frail D. A., 2017, A\&A, 598, A78

James C. W., Anderson G. E., Wen L., Bosveld J., Chu Q., Kovalam M., Slaven-Blair T. J., Williams A., 2019, MNRAS, 489, L75

Kaplan D. L. et al., 2015, ApJ, 814, L25

Kaplan D. L., Murphy T., Rowlinson A., Croft S. D., Wayth R. B., Trott C. M., 2016, PASA, 33, e050

Kaplan D., Sokolowski M., Wayth R., Murphy T., Dobie D., Lynch C., Bannister K., 2017a, GCN, 21637

Kaplan D., Brown I., Sokolowski M., Wayth R., Murphy T., Dobie D., Lynch C., Bannister K., 2017b, GCN, 21927

Kasliwal M. M. et al., 2017, Science, 358, 1559

Lamb G. P. et al., 2019, ApJ, 870, L15

LIGO Scientific Collaboration et al., 2015, Class. Quantum Grav., 32,074001

Margutti R. et al., 2018, ApJ, 856, L18

Mechev A., Oonk J. B. R., Danezi A., Shimwell T. W., Schrijvers C., Intema H., Plaat A., Röttgering H. J. A., 2017, 
Proc. Sci. Vol. 293, Int. Symp. on Grids and Clouds (ISGC), PoS(ISGC2017)002

Metzger B. D., Thompson T. A., Quataert E., 2018, ApJ, 856, 101

Mohan N., Rafferty D., 2015, ascl.soft, ascl:1502.007

Mooley K. P. et al., 2018a, Nature, 554, 207

Mooley K. P. et al., 2018b, Nature, 561, 355

Mooley K. P. et al., 2018c, ApJ, 868, L11

Nakar E., Piran T., 2011, Nature, 478, 82

Nissanke S., Kasliwal M., Georgieva A., 2013, ApJ, 767, 124

Obenberger K. S. et al., 2014, ApJ, 785, 27

Ochsenbein F., Bauer P., Marcout J., 2000, A\&AS, 143, 23

Offringa A. R., de Bruyn A. G., Biehl M., Zaroubi S., Bernardi G., Pandey V. N., 2010, MNRAS, 405, 155

Offringa A. R., van de Gronde J. J., Roerdink J. B. T. M., 2012a, A\&A, 539, A95

Offringa A. R., de Bruyn A. G., Zaroubi S., 2012b, MNRAS, 422, 563

Oliphant T., 2006, Guide to NumPy. USA: Trelgol Publishing

Pandey V. N., van Zwieten J. E., de Bruyn A. G., Nijboer R., 2009, in Saikia D. J., Green D. A., Gupta Y., Venturi T., eds, ASP Conf. Ser. Vol. 407, The Low-Frequency Radio Universe. Astron. Soc. Pac., San Francisco, p. 384

Piro L. et al., 2019, MNRAS, 483, 1912

Radice D., Perego A., Hotokezaka K., Fromm S. A., Bernuzzi S., Roberts L. F., 2018a, ApJ, 869, 130

Radice D., Perego A., Bernuzzi S., Zhang B., 2018b, MNRAS, 481,3670

Resmi L. et al., 2018, ApJ, 867, 57

Rowlinson A., Anderson G. E., 2019, MNRAS, 489, 3316

Rowlinson A. et al., 2019, MNRAS, 490, 3483

Scaife A. M. M., Heald G. H., 2012, MNRAS, 423, L30

Shimwell T. W. et al., 2017, A\&A, 598, A104

Shimwell T. W. et al., 2019, A\&A, 622, A1

Smirnov O. M., Tasse C., 2015, MNRAS, 449, 2668

Swarup G., 1991, in Cornwell T. J., Perley R. A., eds, ASP Conf. Ser. Vol. 19, Radio Interferometry: Theory, Techniques, and Applications, IAU Colloquium 131. Astron. Soc. Pac., San Francisco, p. 376

Tasse C., 2014a, A\&A, 566, A127

Tasse C., 2014b, preprint (arXiv:1410.8706)

Tasse C. et al., 2018, A\&A, 611, A87

Taylor M. B., 2005, in Shopbell P., Britton M., Ebert R., eds, ASP Conf. Ser. Vol. 347, Astronomical Data Analysis Software and Systems XIV. Astron. Soc. Pac., San Francisco, p. 29

Tingay S. J. et al., 2013, PASA, 30, e007

Troja E. et al., 2017, Nature, 551, 71

Troja E. et al., 2018, MNRAS, 478, L18

Troja E. et al., 2019, MNRAS, 489, 1919

van Diepen G., Dijkema T. J., 2018, ascl.soft, ascl:1804.003

van Haarlem M. P. et al., 2013, A\&A, 556, A2

van Weeren R. J. et al., 2016, ApJS, 223, 2

Williams W. L. et al., 2016, MNRAS, 460, 2385

Wilson W. E. et al., 2011, MNRAS, 416, 832

Yancey C. C. et al., 2015, ApJ, 812, 168

Yang Y. Y. et al., 2019, New Astron., 70, 51

Yu Y.-W., Liu L.-D., Dai Z.-G., 2018, ApJ, 861, 114

${ }^{1}$ ASTRON, the Netherlands Institute for Radio Astronomy, Oude Hoogeveensedijk 4, 7991 PD Dwingeloo, The Netherlands

${ }^{2}$ International Centre for Radio Astronomy Research, Curtin University, GPO Box U1987, Perth, WA 6845, Australia

${ }^{3}$ Leiden Observatory, Leiden University, PO Box 9513, 2300 RA, Leiden, The Netherlands

${ }^{4}$ Anton Pannekoek Institute for Astronomy, University of
Amsterdam, Science Park 904, 1098 XH Amsterdam, The Netherlands

${ }^{5}$ GRAPPA, Anton Pannekoek Institute for Astronomy and Institute of High-Energy Physics, University of Amsterdam, Science Park 904, 1098 XH Amsterdam, The Netherlands ${ }^{6}$ Nikhef, Science Park 105, 1098 XG Amsterdam, The Netherlands

${ }^{7}$ Department of Astrophysical Sciences, Peyton Hall, Princeton University, Princeton, NJ 08544, USA

${ }^{8}$ Research Center for the Early Universe, Graduate School of Science, University of Tokyo, Bunkyo-ku, Tokyo 1130033, Japan

${ }^{9}$ SRON Netherlands Institute for Space Research, Sorbonnelaan 2, 3584 CA, Utrecht, The Netherlands

${ }^{10}$ Department of Astrophysics/IMAPP, Radboud University Nijmegen, PO Box 9010, 6500 GL Nijmegen, The Netherlands

${ }^{11}$ GEPI and USN, Observatoire de Paris, Université PSL, CNRS, 5 Place Jules Janssen, 92190 Meudon, France

${ }^{12}$ Department of Physics and Electronics, Rhodes University, PO Box 94, Grahamstown, 6140, South Africa

${ }^{13}$ Centre for Astrophysics Research, School of Physics, Astronomy and Mathematics, University of Hertfordshire, College Lane, Hatfield AL10 9AB, UK

${ }^{14}$ SURFsara, PO Box 94613, 1090 GP Amsterdam, The Netherlands

${ }^{15}$ Astrophysics, Department of Physics, University of Oxford, Keble Road, Oxford OX1 3RH, UK

${ }^{16}$ Sydney Institute for Astronomy, School of Physics, The University of Sydney, NSW 2006, Australia

${ }^{17}$ CSIRO Astronomy and Space Science, PO Box 76, Epping NSW 1710, Australia

${ }^{18}$ Department of Physics, University of Virginia, PO Box 400714 Charlottesville, Virginia 22904-4714, USA

${ }^{19}$ Institute of Astronomy, University of Cambridge, Madingley Road, Cambridge CB3 OHA, UK

${ }^{20}$ University of Technology Sydney, 15 Broadway, Ultimo NSW 2007, Australia

${ }^{21}$ University of the Virgin Islands, 2 Brewers Bay Road, Charlotte Amalie, USVI 00802, USA

${ }^{22}$ Laboratoire AIM (CEA/IRFU - CNRS/INSU - Université Paris Diderot), F-91191 Gif-sur-Yvette, France

${ }^{23}$ Station de Radioastronomie de Nançay, Observatoire de Paris, PSL Research University, CNRS, Univ. Orléans, OSUC, 18330 Nançay, France

${ }^{24}$ Thüringer Landessternwarte, Sternwarte 5, D-07778 Tautenburg, Germany

${ }^{25}$ LPC2E - Université d'Orléans / CNRS, 45071 Orléans cedex 2, France

${ }^{26}$ SKA Organisation, Jodrell Bank Observatory, SK11 9DL, $U K$

${ }^{27}$ Cahill Center for Astronomy and Astrophysics, California Institute of Technology, Pasadena, CA, USA

${ }^{28}$ Instituto de Astrofísica de Canarias, 38205 La Laguna, Tenerife, Spain

${ }^{29}$ Departamento de Astrofísica, Universidad de La Laguna, E-38206 La Laguna, Tenerife, Spain

${ }^{30}$ South African Radio Astronomy Observatory, 2 Fir Street, Black River Park, Observatory, 7925, South Africa

${ }^{31}$ Department of Physics and Astronomy, University of the Western Cape, Cape Town 7535, South Africa

${ }^{32}$ Department of Physics, The George Washington Univer- 
sity, 725 21st Street NW, Washington, DC 20052, USA

${ }^{33}$ Astronomy, Physics, and Statistics Institute of Sciences (APSIS), The George Washington University, Washington, DC 20052, USA

${ }^{34}$ LESIA and USN, Observatoire de Paris, CNRS, PSL, SU/UP/UO, 92195 Meudon, France

${ }^{35}$ Technical University of Berlin, Institute of Geodesy and Geoinformation Science, Faculty VI sec. H 12, Main Building Room H 5121, Straße des 17. Juni 135, 10623 Berlin, Germany

${ }^{36}$ GFZ German Research Centre for Geosciences, Telegrafenberg, 14473 Potsdam, Germany

${ }^{37}$ Eindhoven University of Technology, PO Box 513, 5600 $M B$ Eindhoven, The Netherlands

${ }^{38}$ Kapteyn Astronomical Institute, University of Groningen, PO Box 800, 9700 AV Groningen, The Netherlands

${ }^{39}$ Hamburger Sternwarte, University of Hamburg, Gojenbergsweg 112, D-21029 Hamburg, Germany

${ }^{40}$ Max Planck Institute for Astrophysics, KarlSchwarzschild-Str. 1, 85748 Garching, Germany

${ }^{41}$ Jodrell Bank Centre for Astrophysics, Alan Turing Building, Department of Physics and Astronomy, The University of Manchester, Oxford Road, Manchester M13 9PL, UK

${ }^{42}$ Vrije Universiteit Brussel, Department of Physics and Astronomy, B-1050 Brussels, Belgium

${ }^{43}$ Astronomisches Institut der Ruhr-Universität Bochum, Universitätsstraße 150, 44780 Bochum, Germany

${ }^{44}$ Space Radio-Diagnostics Research Centre, University of Warmia and Mazury in Olsztyn, Prawocheńskiego 9, 10-720 Olsztyn, Poland

${ }^{45}$ Leibniz-Institut für Astrophysik Potsdam (AIP), An der Sternwarte 16, D-14482 Potsdam, Germany

${ }^{46}$ Deutsches Elektronen-Synchrotron (DESY), 15738

Zeuthen, Germany

${ }^{47}$ Erlangen Centre for Astroparticle Physics (ECAP), Friedrich-Alexander-Universität Erlangen-Nürnberg, 91058 Erlangen, Germany

${ }^{48}$ Center for Information Technology (CIT), University of Groningen, Groningen, The Netherlands

${ }^{49}$ Univ. Lyon, Univ. Lyon1, Ens de Lyon, CNRS, Centre de Recherche Astrophysique de Lyon UMR5574, 9 av. Charles André F-69230, Saint-Genis-Laval, France

${ }^{50}$ Poznań Supercomputing and Networking Center (PCSS), Poznań, Poland

${ }^{51}$ Max-Planck-Institut für Radioastronomie, Auf dem Hügel 69, 53121 Bonn, Germany

${ }^{52}$ Fakultät für Physik, Universität Bielefeld, Postfach 100131, 33501 Bielefeld, Germany

${ }^{53}$ Jagiellonian University, Astronomical Observatory, ul. Orla 171, 30-244 Kraków, Poland

${ }^{54}$ Department of Space, Earth and Environment, Chalmers University of Technology, Onsala Space Observatory, SE43992 Onsala, Sweden

This paper has been typeset from a $\mathrm{T}_{\mathrm{E}} \mathrm{X} / \mathrm{LAT}_{\mathrm{E}} \mathrm{X}$ file prepared by the author. 